\title{
The current value of quantitative shear wave sonoelastography in parotid gland tumors
}

\author{
Vivian Thimsen ${ }^{1}$, Miguel Goncalves ${ }^{2}$, Michael Koch $^{1}$, Konstantinos Mantsopoulos ${ }^{1}$, Joachim Hornung ${ }^{1}$, \\ Heinrich Iro ${ }^{1}$, Mirco Schapher ${ }^{1}$ \\ ${ }^{1}$ Department of Otorhinolaryngology, Head and Neck Surgery, Universitätsklinikum Erlangen, University of Erlangen-Nürnberg (FAU), Erlangen, \\ Germany; ${ }^{2}$ Department of Otorhinolaryngology, Head and Neck Surgery, University of Aachen, RWTH, Aachen, Germany \\ Contributions: (I) Conception and design: M Schapher, V Thimsen; (II) Administrative support: M Schapher, V Thimsen, M Koch, K Mantsopoulos; \\ (III) Provision of study materials or patients: H Iro, M Koch, J Hornung, K Mantsopoulos; (IV) Collection and assembly of data: V Thimsen, M \\ Schapher, M Goncalves; (V) Data analysis and interpretation: V Thimsen, M Schapher, M Goncalves; (VI) Manuscript writing: All authors; (VII) \\ Final approval of manuscript: All authors. \\ Correspondence to: Mirco Schapher, MD, PhD, MSc. Department of Otorhinolaryngology, Head and Neck Surgery, Universitätsklinikum Erlangen- \\ Nürnberg, University of Erlangen-Nürnberg (FAU), Waldstrasse 1, 91054 Erlangen, Germany. Email: mirco.schapher@uk-erlangen.de.
}

Background: The preoperative differentiation between salivary gland tumor entities using computed tomography, magnetic resonance imaging (MRI) and ultrasound (US) is still limited. Biopsies are often regarded as indispensable for properly characterizing these various lesions. The aim of this study was to analyze the value of acoustic radiation force impulse (ARFI) sonoelastography as an US differentiation tool when examining parotid gland (PG) lesions.

Methods: We included 104 patients with PG masses in this study, employing two different US devices using quantitative ARFI-sonoelastography (Siemens Acuson-S3000, n=59; Siemens Acuson-Sequoia, n=45).

The ability of sonoelastographic measurements to differentiate between different neoplasms was compared and analyzed for both US machines.

Results: Quantitative shear wave sonoelastography is limited in its ability to reliably differentiate between tumor entities of the PG as a stand-alone parameter. Measurement results were unsystematically distributed and not transferable between the two US devices. A significant differentiation of benign and malignant lesions was not possible with either US machine ( 33000 : $\mathrm{P}=0.770$, Sequoia: $\mathrm{P}=0.382$ ). A differentiation between pleomorphic adenomas (PA) and Warthin tumors was only possible with the Acuson S3000 system ( $\mathrm{P}=0.001$, Spearman-Rho $=0.492$, sensitivity $73.9 \%$, specificity $65.0 \%)$.

Conclusions: A reliable identification and differentiation of PG tumors as well as clinical treatment decisions cannot be made with the sole use of ARFI-sonoelastography. The results emphasize the devicedependence and high error-proneness of this US technique when examining lesions of the PG.

Keywords: Ultrasound (US); acoustic radiation force impulse (ARFI); sonoelastography; parotid gland (PG); salivary gland tumors

Submitted Nov 17, 2020. Accepted for publication Feb 04, 2021.

doi: $10.21037 / g s-20-837$

View this article at: http://dx.doi.org/10.21037/gs-20-837

\section{Introduction}

Salivary gland tumors represent about $2-6 \%$ of all head and neck neoplasms (1-3), the majority arising within the parotid gland (PG). Benign lesions constitute $80-90 \%$ and malignant lesions $10-20 \%$ of all cases (2). The WHO has listed 11 benign and 22 malignant salivary gland tumor entities (4), a considerable number which poses a diagnostic and therapeutic challenge.

Computed tomography (CT) and magnetic resonance 
imaging (MRI) are the most frequently used imaging methods for assessing these masses (5-7). Unfortunately, overlapping characteristics of the various tumor entities reduce the validity of these imaging techniques to distinguish different neoplasms from one another. Former studies evaluating CT and MRI modalities to predict the nature of PG neoplasms reported on limited sensitivity and specificity $(8,9)$. Although recent MRI techniques considering diffusion and perfusion patterns have occasionally been described as helpful, these are expensive, time-consuming and still limited in their overall conclusiveness $(8,10,11)$. Consequently, biopsies are often still regarded as indispensable for a proper characterization of lesions and further therapeutic planning (12). However, the course of the facial nerve within the gland and the risk of its injury performing invasive diagnostic measures explain the continuing desire for further non-invasive diagnostic options.

Ultrasound (US) is an additional valuable tool to assess salivary gland lesions and supplies complementary information. It is fast, easily obtainable, radiation-free and cost-effective. Conventional B-mode and colorDoppler examinations allow a precise evaluation of certain characteristics of salivary gland masses but cannot-each or in combination-reliably differentiate between the different tumor entities or at least between benign and malignant lesions (8,9,13-16). At present, extended US techniques are required to answer these remaining questions.

The benefit of sonoelastographic measurements to further characterize neoplasms is accepted in breast (17), thyroid (18-20), liver $(21,22)$ and prostate lesions $(23)$. Different elastography techniques are available all of which assess tissue stiffness/elasticity as a potential indicator of malignancy. Although malignant tumors often proved to be stiffer than benign lesions in the before mentioned organs, several studies came to different conclusions when analyzing salivary gland tumors. This reported discrepancy might be due to diverse sonoelastographic measurement techniques and different devices used in past studies assessing salivary glands, limiting the overall comparability of the results. For sonoelastography in salivary gland tumors, large differences were reported in specificity, ranging from 26-89\% (15,24-29). A recent meta-analysis (30) concluded that quantitative and semi-quantitative methods performed better than qualitative ones, and that the sonoelastographic measurement technique was the most significant factor to cause the heterogeneity of former study results, diluting the informative value.

We designed the present study to evaluate the use and reliability of elastographic measurements in PG tumors, excluding the influence of other US features to avoid any form of bias. Only quantitative methods (ARFI-VTIQ shear wave technique and ARFI-2D shear wave technique) were employed, using two different high-end US devices. To the best of our knowledge, this is the first assessment of this kind.

We investigated whether it was possible to reliably differentiate between the various tumor entities or between benign and malignant masses using quantitative shear wave elastography as an independent parameter. Adhering to a strict examination protocol, we further analyzed whether obtained measurement values were dependent on the US device or the shear wave detection technique applied, whether the values were stable and transferable/comparable, and whether potential cut-off values could be established to support the diagnostic process in PG neoplasms.

We present the following article in accordance with the MDAR reporting checklist (available at http://dx.doi. org/10.21037/gs-20-837).

\section{Methods}

\section{Study population}

Data were obtained at an academic tertiary referral center specializing in salivary gland pathologies. Written informed consent was obtained from each patient for diagnostic procedures, therapeutic measures and scientific data processing, approved by the University's ethical review board (IRB No. 156_20 Bc), observing the university's general contract conditions and the World Medical Association Declaration of Helsinki (as revised in 2013).

In total, we included 104 patients in the study cohort retrospectively [ 43 men (41.3\%), 61 women (58.7\%); mean age $54.9 \pm 15.6$ years, Table 1] who presented to our department with a PG mass from May 2016 to September 2019. Every patient received a clinical and US examination prior to surgery, all of which were carried out in our department. Individuals were excluded $(n=17)$ if the neoplasm could not be depicted completely on US (tumor size, dorsal sound extinction caused by the mandible), or if they did not receive further surgical treatment in our department, including a histopathological analysis of the neoplasm after its complete removal. We did not consider results of core needle biopsies. All tumors were examined 
Table 1 Study population and observed tumor entities

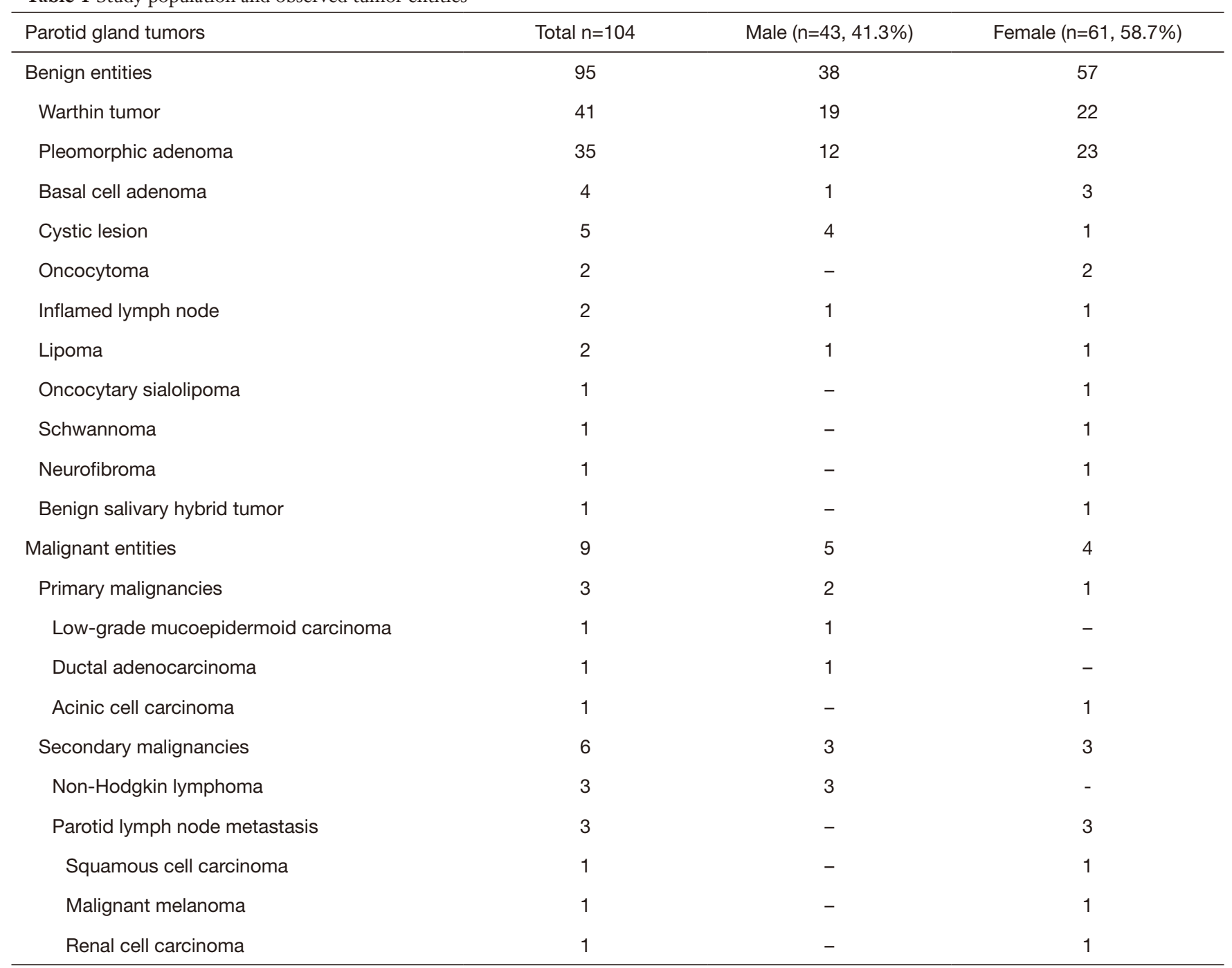

and classified by experienced pathologists of the university specializing in salivary gland diseases.

\section{US examinations}

We performed US examinations using two different highend US devices [Acuson S3000, 9L4 transducer, ARFIVTIQ (Virtual Touch Imaging and Quantification) shear wave elastography; Acuson Sequoia, 10L4 transducer, ARFI-2D-shear wave elastography; all products from Siemens Healthineers, Erlangen, Germany; ARFI: Acoustic Radiation Force Impulse]. All patients received an US examination of the head and neck, including the PG. US was performed by three otolaryngologists, all certified by the German society of US in medicine (DEGUM), and with several years of experience in the treatment of salivary gland diseases. The standardized examination protocol included a conventional B-scan, color-coded duplex sonography and elastographic measurements, with precise definitions of how the elastographic recordings had to be performed. Color gain and pulse repetition frequencies were adapted as necessary to avoid artifacts. We determined tumor sizes on the axial and coronal axes and recorded still pictures and video sequences to reduce examiner-dependency. As US cannot depict the facial nerve appropriately, we did not address the spatial relationship between the tumors and the 
nerve in this study.

For the elastographic measurements of tumors, the transducers were positioned directly above the mass to ensure a perpendicular measurement angle, held with only two fingers so that the mere weight of the transducer was responsible for any pre-compression of the tissue. A sufficient quantity of US gel (Aquasonic 100, Parker Laboratories, Fairfield, USA) was applied and sufficient contact with the skin was ensured. We adjusted the imaging depths to depict the neoplasm completely. Each system allowed the tracking of shear waves within the region of interest (ROI) perpendicular to the transmitted longitudinal push pulse, and time-to-peak-analysis served to obtain shear wave velocities $[\mathrm{SWV},(\mathrm{m} / \mathrm{s})]$. Each tumor was examined with one US device only, as both of the devices were not available simultaneously.

With the S3000 system ( $=59$ ), we obtained elastographic measurements within a rectangular ROI. The window was placed to center the tumor, the size adjusted to completely cover the tumor area, including a rim of healthy adjacent tissue, and the measurement record was started. Within the ROI, we defined independent measurement points exclusively within the neoplastic tissue respecting $2-3 \mathrm{~mm}$ distance from each other, arranged in rows and columns, to ensure a homogeneous distribution throughout the tumor, resulting in 4-18 record values for each lesion, dependent on its size. SWVs $[\mathrm{m} / \mathrm{s}]$ including their individual depths $[\mathrm{cm}]$ and the quality of each measurement were recorded (Figure $1 A, B, C$ ). The mean value of the SWVs was obtained ( \pm standard deviation, $\mathrm{SD}$ ) for each neoplasm.

With the Sequoia system $(n=45)$, we performed elastographic measurements after defining a circular ROI exclusively covering the tumor. The software calculated the mean values of the $\mathrm{SWVs}[\mathrm{m} / \mathrm{s}]$, modulus of elasticities [E, $(\mathrm{kPa})]$ and SD within this ROI. In addition, we recorded the ROI mean depth, the ROI diameter and the quality of every measurement (Figure 1D,E,F). As in the S3000, we used the mean SWV-values ( \pm standard deviation, $\mathrm{SD}$ ) for further analysis.

\section{Statistical analysis}

According to the histopathological results, matching entities were categorized in groups. We calculated significances, specificities, correlation coefficients, ROCcurves and possible cut-off-values for each specific question (SPSS, Version 24, IBM Corporation, Armonk, USA). As a Gaussian distribution of the SWV-values could not be assumed (Kolmogorov-Smirnov and Shapiro-Wilks test), we applied the Mann-Whitney test for further data analysis. $P$ values $<0.05$ were considered significant.

\section{Results}

\section{Study population and observed tumor entities}

US examinations and ARFI-shear wave sonoelastographic measurements were performed in 104 patients with PG masses (Table 1). After surgery, pathohistological analyses determined $91.3 \%$ of the tumors as benign [ $n=95 / 104$; 41 Warthin tumors (WT), 35 pleomorphic adenomas (PA), 4 basal cell adenomas, 5 lymphoepithelial cysts, 2 oncocytomas, 2 inflammatory lymphnodes, 2 lipomas, 1 oncocytary sialolipoma, 1 schwannoma, 1 neurofibroma and 1 benign salivary hybrid tumor] and $8.7 \%$ as malignant $[n=9 / 104 ; 3$ primary salivary gland malignomas (1 low-grade mucoepidermoid carcinoma, 1 ductal adenocarcinoma and 1 acinic cell carcinoma without highgrade transformation), 6 secondary malignancies of the PG (3 non-Hodgkin-lymphomas, 3 lymphnode metastases of squamous cell carcinoma, malign melanoma, renal cell carcinoma)].

\section{Absolute SWV-values obtained by ARFI-2D shear wave sonoelastography were not transferable between the different US systems}

To examine the constancy of SWV-values using the ARFIshear wave technique, we compared the SWVs of different tumor subgroups obtained with the two different machines (Table 2). SWV-values for identical tumor entities differed more between the two devices than they did between the various tumor entities obtained with the same device (e.g., the differences in SWV-values between PA when comparing the S3000 with the Sequoia were larger than the differences in SWV-values between PA and Warthin tumors within each of the two devices, Table 3). Various calculations to test whether a correlation (e.g., a potential conversion factor) existed between the mean SWV-values and the employed US device revealed that the measurement values were unsystematically distributed in both machines and therefore not transferable from one system to the other (significance tests, quotient formation, subtraction of mean values; not shown). As the mean SWV-values were at least systemdependent, further statistics had to be calculated for both US systems separately. 

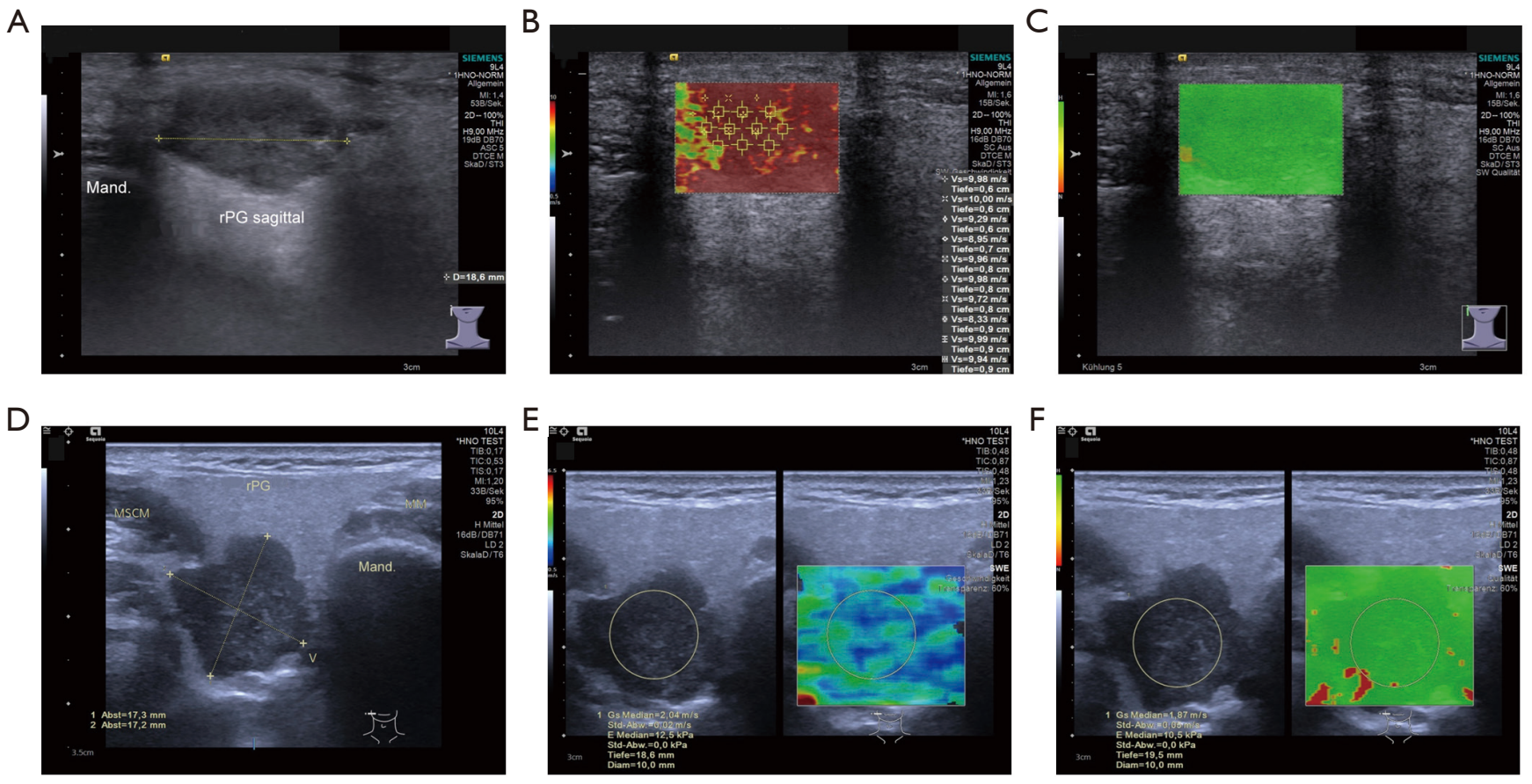

Figure 1 Ultrasonography in parotid gland tumors, using either the Acuson S3000 (A-C) or the Acuson Sequoia (D-F) ultrasound system. (A) B-mode image of the right PG, depicting a mass within the parenchyma as a hypoechoic, rounded and quite homogeneous structure with defined margins. The histopathological examination subsequently identified this lesion as a pleomorphic adenoma. (B) Quantitative ARFI (Acoustic Radiation Force Impulse) shear wave elastography (VTIQ: Virtual Touch Imaging and Quantification) measurement of the lesion depicted in (A). The quadrangular region of interest (ROI) in which SWVs were obtained was positioned to cover the tumor. After the record was taken, in this case ten different measurement values were determined exclusively within the tumorous area. SWVs are additionally shown as color-coded signals (red: high velocities; green: low velocities). (C) Quality assurance of the obtained elastographic record revealing a high and homogeneous measurement quality throughout (green). (D) B-mode image of a second right PG in a different patient. The hypoechoic lesion here exhibits well-defined boundaries, a polycyclic shape and a slightly heterogeneous interior, also representing a pleomorphic adenoma. (E) Quantitative ARFI-2D-shear wave elastography of the mass depicted in (D). The Sequoia software uses a circular ROI within which SWVs were averaged automatically. Velocities are additionally shown as color-coded signals (red: high velocities; green: intermediate velocities; blue: low velocities). (F) Elastographic quality-assessment of the obtained image. Besides the color-coded representation, the system software automatically calculated and displayed the standard deviation (Std-Abw.) of the obtained measurement values. All parameters revealed a consistent high and homogeneous image quality. rPG, right parotid gland; MM, masseter muscle; Mand, mandible; V, retromandibular vein; MSCM, sternocleidomastoid muscle; SWV, shear wave velocity.

Table 2 Mean values for shear wave velocities (SWVs) in different parotid gland lesions, obtained with two different US devices

\begin{tabular}{|c|c|c|c|c|c|c|}
\hline Tumor entities & Total & S3000, n & SWVs [m/s] S3000 & Sequoia, $\mathrm{n}$ & SWVs [m/s] Sequoia & $\mathrm{P}$ \\
\hline Pleomorphic adenoma & 35 & 23 & $5.95 \pm 1.92$ & 12 & $3.16 \pm 1.14$ & 0.000 \\
\hline Warthin tumors & 41 & 20 & $4.13 \pm 1.00$ & 21 & $2.42 \pm 0.69$ & 0.000 \\
\hline Other benign tumors & 19 & 9 & $3.07 \pm 1.97$ & 10 & $2.79 \pm 0.85$ & 0.227 \\
\hline Total & 104 & 59 & $4.88 \pm 1.88$ & 45 & $2.68 \pm 0.9$ & - \\
\hline
\end{tabular}

$P$ values indicate significant differences in obtained SWV-values when comparing the two devices. 
Table 3 Tumor volumes, shear wave velocity (SWV)-values and correlations obtained from pleomorphic adenomas (PA) and Warthin tumors (WT) with two different ultrasound (US) devices (Acuson S3000 and Acuson Sequoia)

\begin{tabular}{|c|c|c|c|c|}
\hline & \multicolumn{2}{|c|}{ PA } & \multicolumn{2}{|c|}{ WT } \\
\hline$n$ & 21 & 12 & 20 & 20 \\
\hline Mean tumor volume $\left[\mathrm{cm}^{3}\right]$ & $3.84 \pm 4.70$ & $4.14 \pm 3.47$ & $6.72 \pm 6.75$ & $5.70 \pm 5.37$ \\
\hline $\mathrm{p}_{\text {Volume }}$ & \multicolumn{2}{|c|}{0.818} & \multicolumn{2}{|c|}{0.529} \\
\hline$P_{\text {swv }}$ & \multicolumn{2}{|c|}{0.000} & \multicolumn{2}{|c|}{0.000} \\
\hline Spearman-Rho $_{\text {swv-vol }}$ & 0.062 & 0.284 & 0.259 & 0.002 \\
\hline
\end{tabular}

The mean tumor volumes did not differ significantly between both US devices $\left(P_{\text {Volume }}\right)$, but the mean SWV-values did $\left(P_{\text {swv }}\right)$. No significant correlation between tumor volumes and SWV-values was detected. PA, pleomorphic adenomas; WT, Warthin tumors; SWV, shear wave velocity.

\section{PG tumor dignities and entities could not be differentiated reliably by ARFI-shear wave sonoelastography}

We calculated the results separately for each of the two US devices. In benign tumors, the mean $\mathrm{SWV}$-values were $4.88 \pm 1.88 \mathrm{~m} / \mathrm{s}(\mathrm{S} 3000)$ and $2.71 \pm 0.91 \mathrm{~m} / \mathrm{s}$ (Sequoia), revealing significant differences between both machines $\left(\mathrm{P}_{\mathrm{BEN}}=0.000\right)$. For malignant lesions, the $\mathrm{SWV}$-values were $5.04 \pm 1.94 \mathrm{~m} / \mathrm{s}(\mathrm{S} 3000)$ and $2.20 \pm 0.77 \mathrm{~m} / \mathrm{s}$ (Sequoia), also detecting significant differences $\left(\mathrm{P}_{\mathrm{MAL}}=0.026\right)$ when the two systems were compared (Table 2).

When we compared SWVs of benign with those of malignant lesions for each US system separately, the statistical results indicated that it was neither possible with the $\mathrm{S} 3000(\mathrm{P}=0.770)$ nor with the Sequoia system $(\mathrm{P}=0.382)$ to detect significant differences (Figure $2 A, B$ ). SpearmanRho did not show any significant correlation between SWVs and tumor dignity in any of the two US devices used ${\text { (Spearman- } \text { Rho }_{S 3000}=0.040 \text {; Spearman-Rho }}_{\text {Sequoia }}=0.133$ ). The receiver operating characteristic (ROC) indicated a random value distribution for benign and malignant lesions in both devices $\left(\mathrm{AUC}_{\mathrm{S} 3000} 0.536 ; \mathrm{AUC}_{\text {Sequoia }}=0.315\right)$.

Second, we asked whether these statistically insignificant results were caused by varying and potentially disturbing SWVs obtained from PA, as the latter occur in different subtypes with fluctuating consistencies $(31,32)$. To assess this, we firstly calculated the SWV-values of all benign PG lesions except PA for each US device separately (Figure 2C,D; mean $\mathrm{SWV}_{\mathrm{S} 3000-\mathrm{BENwoPA}}=3.98 \pm 1.35 \mathrm{~m} / \mathrm{s}$; mean $\mathrm{SWV}_{\text {Sequoia- }}$ BENwoPA $=2.53 \pm 0.75 \mathrm{~m} / \mathrm{s}$ ) and compared them with those of malignancies (mean $\mathrm{SWV}_{\mathrm{S} 3000-\mathrm{MAL}}=5.04 \pm 1.94 \mathrm{~m} / \mathrm{s}$; mean $\left.S W V_{\text {Sequoia-MAL }}=2.20 \pm 0.77 \mathrm{~m} / \mathrm{s}\right)$. For both devices, we neither found significant differences $\left(\mathrm{p}_{\mathrm{S} 3000}=0.162 ; \mathrm{p}_{\text {Sequoia }}=0.483\right)$, nor did Spearman-Rho indicate any correlation between SWVs and tumor dignity (Spearman-Rho $\mathrm{S}_{3000}=0.240$; Spearman$\mathrm{Rho}_{\text {Sequia }}=0.126$; ROC-curves). In conclusion, the wide range of SWVs obtained from PA did not influence the mean SWV-values of other benign entities in a way that impeded their differentiation from malignant lesions.

Third, we compared the SWVs of PA exclusively (mean $\mathrm{SWV}_{\mathrm{S} 3000-\mathrm{PA}}=5.95 \pm 1.92 \mathrm{~m} / \mathrm{s}$; mean $\mathrm{SWV}_{\text {Sequoia }-\mathrm{PA}}=3.16 \pm$ $1.14 \mathrm{~m} / \mathrm{s}$ ) with those of all other, grouped benign PG tumors except PA (Figure $2 E, F$; mean $\mathrm{SWV}_{\mathrm{S} 3000-\mathrm{BENwoPA}}=3.98 \pm 1.35$ $\mathrm{m} / \mathrm{s}$; mean $\mathrm{SWV}_{\text {Sequoia-BENwoPA }}=2.53 \pm 0.75 \mathrm{~m} / \mathrm{s}$ ). Here we achieved varying results: SWV-values obtained with the S3000 differed significantly between the two groups (PA vs. all other grouped benign tumors, $\mathrm{P}=0.000$; SpearmanRho $=0.517$, cut - off value $=4.39 \mathrm{~m} / \mathrm{s}$; sensitivity: $82.6 \%$, specificity: $72.4 \%$ ), whereas this was not the case for values obtained with the Sequoia $(\mathrm{P}=0.072$; Spearman-Rho $=0.281$; ROC-curves).

Fourth, we compared the mean SWVs of PA with those of malignant lesions, yielding no statistically significant differences in any system (Figure 2G,H; S3000: $\mathrm{P}=0.270$, Spearman-Rho $=0.277$; Sequoia: $\mathrm{P}=0.234$, Spearman - Rho $=0.249 ;$ ROC-curves).

\section{The ability to differentiate PA from Warthin tumors (WT) was limited and device-dependent}

We posed the hypothetical question whether ARFIshear wave sonoelastography is useful for differentiating between PA and WT, which comprise the two most 

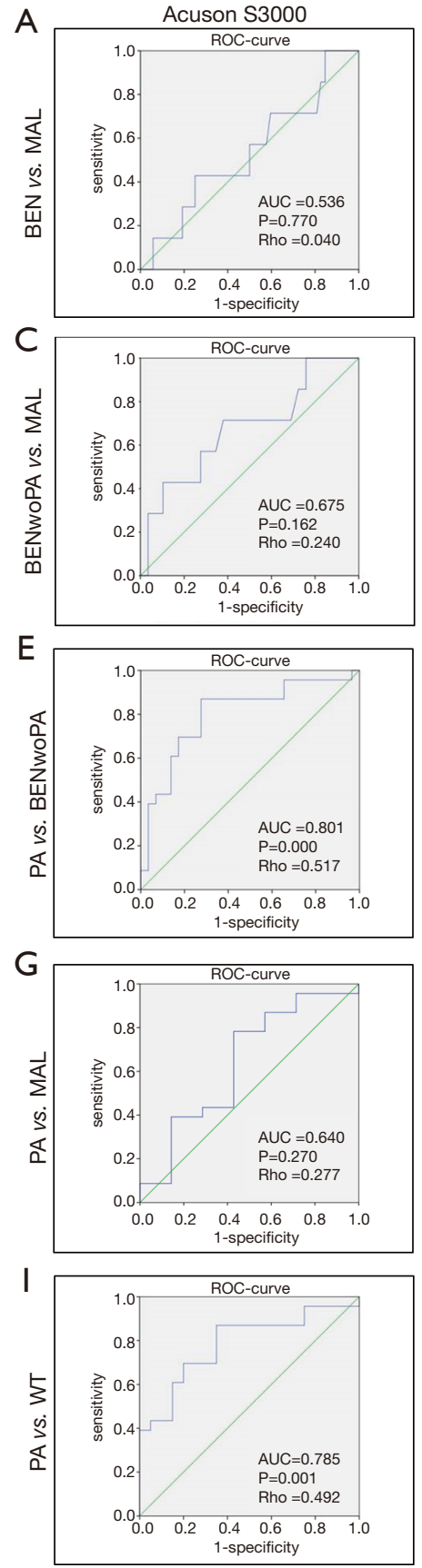
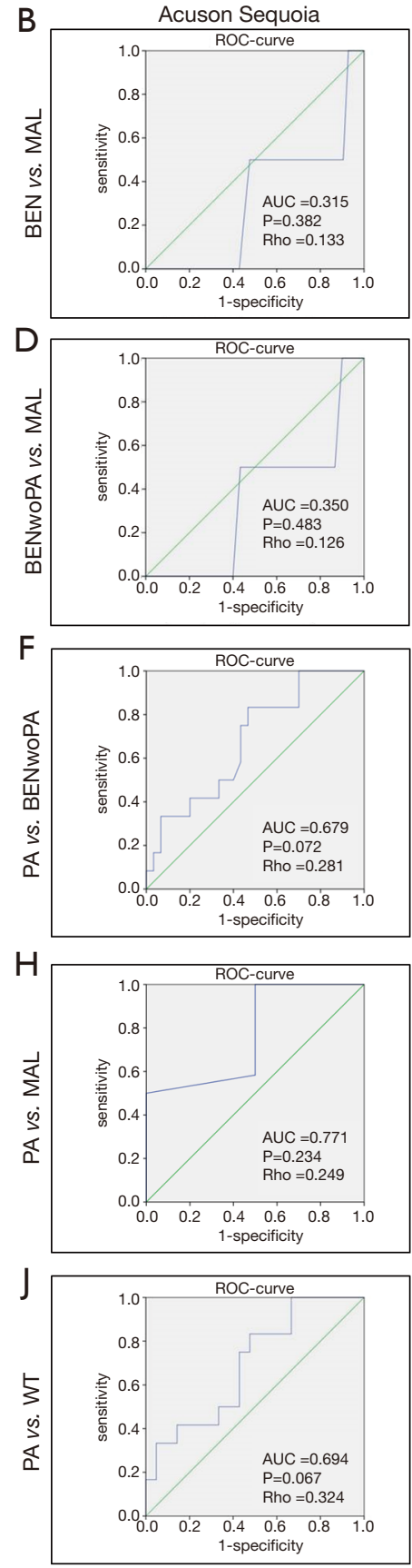

Figure 2 Receiver operating characteristic (ROC) curves for both ultrasound (US) devices in specific attempts at tumor differentiation. The accuracy of shear wave velocity (SWV)-values to distinguish specific tumors from one another is displayed in each image. The left column illustrates the results obtained with the Acuson S3000 system (A,C,E,G,I), the right column those obtained with the Acuson Sequoia (B,D,F,H,J). The course of the curve near the diagonal line indicates a random distribution of the measured values independent of the histopathological nature of the tumors compared. (A,B) Velocity value distribution comparing benign with malignant PG lesions. (C,D) Velocity value distribution comparing benign masses except PA with malignant lesions. (E,F) Velocity value distribution comparing PA with all other benign lesions except PA. $(\mathrm{G}, \mathrm{H})$ Velocity value distribution comparing PA with malignant lesions. (I,J) Velocity value distribution comparing PA and WT. Taken together, we could only detect significant results in the sonoelastographic differentiation of PA from WT $(\mathrm{P}=0.000)$ and the differentiation of $\mathrm{PA}$ from BENwoPA $(\mathrm{P}=0.001)$ using the $\mathrm{S} 3000$. With the Sequoia, we could not detect any significant results. PG, parotid gland; PA, pleomorphic adenomas; WT, Warthin tumors; BENwoPA, benign tumors except PA; BEN, benign tumors; MAL, malignant tumors. 


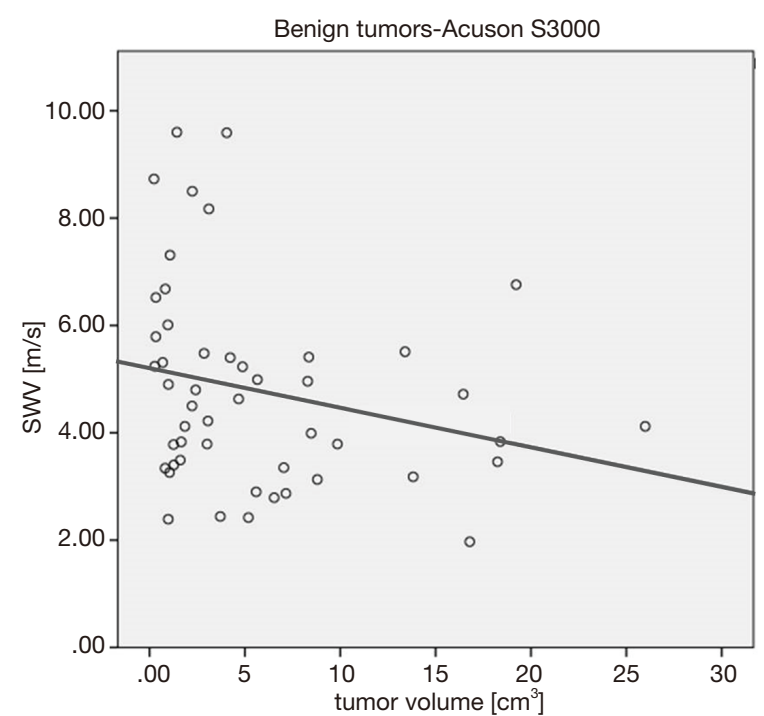

Figure 3 Scatter plots illustrating the correlation of shear wave velocity (SWV-)values and tumor volumes in benign parotid gland (PG) neoplasms (Acuson S3000). A weak, but negative correlation of the recorded the SWV-values and the tumor volume of benign PG tumors was found when the Acuson S3000 US device was used. The wide range of SWV-values for similar tumor volumes is remarkable. The data for the Sequoia are not shown as no significant differences were observed.

common benign lesions of the PG. Statistical evaluations were carried out separately for each system. Using the S3000, the SWV-values of PA differed significantly from those of WT $\left(\mathrm{P}=0.001\right.$; Spearman-Rho $=0.492 ; \mathrm{n}_{\mathrm{PA}}=23$; mean $\mathrm{SWV}_{\mathrm{PA}}=5.95 \pm 1.92 \mathrm{~m} / \mathrm{s} ; \mathrm{n}_{\mathrm{WT}}=20$, mean $\mathrm{SWV}_{\mathrm{WT}}=4.13 \pm$ $1.00 \mathrm{~m} / \mathrm{s}$, Table 2). Here, a SWV-cut-off value to differentiate PA from WT could be defined as $4.62 \mathrm{~m} / \mathrm{s}$ (sensitivity: 73.9\%; specificity: $65.0 \%$ ). Using the Sequoia, no significant discrepancies between PA and WT were found $(\mathrm{P}=0.067)$, only a slight correlation was detected (Spearman-Rho $=0.324$; $\mathrm{n}_{\mathrm{PA}}=12$, mean $\mathrm{SWV} \mathrm{PA}_{\mathrm{PA}}=3.16 \pm 1.14 \mathrm{~m} / \mathrm{s} ; \mathrm{n}_{\mathrm{WT}}=21$, mean $\mathrm{SWV} \mathrm{VT}_{\mathrm{WT}}$ $=2.42 \pm 0.69 \mathrm{~m} / \mathrm{s}$, Table 2). Although SWV-values indicated that PA tended to be generally stiffer than WT in both systems, a cut-off-value could only be determined for one of the two devices (S3000, with a specificity of only $65.0 \%$ ). The ROC curves revealed only a fair degree of accuracy for the differentiation between PA and WT in both systems $\left(\mathrm{AUC}_{\mathrm{S} 3000}=0.785 ; \mathrm{AUC}_{\text {Sequia }}=0.694\right.$, Figure $\left.2 I, 7\right)$.

\section{The tumor size may influence $S W V$-values in the $P G$}

The PG is surrounded by a scarcely elastic fibrous capsule.
An increasing size of a PG neoplasm may therefore increase the compression of the adjacent healthy gland parenchyma which cannot expand due to that capsule, and this might alter the stiffness of the entire gland, influencing SWVvalues. To test this, we correlated the tumor volumes, assuming an ellipsoid body $\left(\frac{4}{3} \pi a b c ; a, b, c\right.$ representing half of the length, width and height of the tumor size) with the SWV-values in each of the two systems. We considered benign lesions only to exclude any influence of tumor infiltration of the adjacent gland and because they comprised the lesions with the largest mean tumor volume in our study cohort.

Surprisingly, we found a significant but weak negative correlation between the tumor volume and SWV-values for lesions examined with the $\mathrm{S} 3000(\mathrm{~N}=49, \mathrm{P}=0.04$, SpearmanRho $=0.295$, mean tumor volume $=5.74 \pm 6.14 \mathrm{~cm}^{3}$, Figure 3), but not for those examined with the Sequoia $(\mathrm{N}=42, \mathrm{P}=0.706$, Spearman $-\mathrm{Rho}=0.060$, mean tumor volume $=4.92 \pm 4.35 \mathrm{~cm}^{3}$; not shown), although the mean tumor volumes did not differ significantly $(\mathrm{P}=0.549)$. When $\mathrm{PA}$ and WT were investigated separately, no correlation between tumor volume and SWV-values for each of the US devices or tumor entities was detected. The recorded tumor volumes did not differ significantly between the two systems $\left(\mathrm{P}_{\mathrm{PA}}=0.818 ; \mathrm{P}_{\mathrm{WT}}=0.529\right)$, while the $\mathrm{SWV}$ values did $\left(\mathrm{P}_{\mathrm{SWv}}=0.000\right.$ each, Table 3$)$. These results again demonstrated the high inconsistency in measurement values, even though two similar US devices were used and similar cases investigated. In summary, we did not obtain congruent results in both machines and could not conclude that larger tumors tend to increase the stiffness of the PG in general (Spearman-Rho $\mathrm{Swv-Vol}_{\text {; }}$ Table 3).

\section{Discussion}

The assessment of PG tumors using US has numerous advantages: US is a fast, cost-effective, radiation free and precise real time imaging modality available nearly anywhere. Treating surgeons can perform the examination on their own, which is particularly helpful during the operation. The further evaluation of cervical lymph nodes complements the overall picture of the lesion. However, bony structures reduce the field of view, and US is limited in depicting tumors extending medially of the mandible, tumors of the deep lobe, the parapharyngeal space or those that are in close contact with the skull base. In these cases, MRI and CT scans can supplement the results of 
US examinations. While CT scans can illustrate bony infiltrations or distant metastasis, MRI is the technique of choice to assess the infiltration of the facial nerve, the dura or the parapharyngeal space. Although US provides sufficient information to plan the surgical procedure without the need for additional imaging in the majority of PG neoplasms, the ability to distinguish different tumor entities is limited in all imaging modalities. This is also true for US, at least if exclusively B-mode or color-Doppler examinations are performed.

The use and benefits of strain elastography and different shear wave elastography techniques in PG lesions have been assessed before, but statements diverged greatly as to whether sonoelastography in general can be considered helpful in identifying PG lesions (15,24-29). A former meta-analysis concluded that quantitative methods-as the ARFI-technique we used in this study-have provided more stable results than semi-quantitative methods (30). As this meta-analysis also considered results obtained with semi quantitative techniques, the summarizing conclusion may have been influenced by these less stable data.

Only scarce data are available on the use of ARFItechniques in PG lesions, obtained in few studies which included only a limited number of patients (29,33-35). These articles evaluated sonoelastography as an add-on method to characterize PG masses, supplemented with other US features. The goal of the present study, including 104 patients, was therefore to evaluate the use and reliability of exclusively quantitative, ARFI-based sonoelastographic measurements in PG tumors.

The different techniques used in US elastography examinations can be confusing. In short, strain elastography techniques detect local changes in tissue displacement, based on the principle that, under compression by an impulse, soft parts of the tissue delocalize more than stiff parts within the ROI. In contrast, shear wave elastography involves a high-frequency US impulse generated by the device to displace the tissue, but the generated shear waves propagate orthogonally to the direction of tissue displacement, with their velocities being recorded and quantified. Additional technical differences exist in the generation of the impulse, the ARFI-technique being one of the options available.

In this context and apart from well-elaborated examination protocols for other organs, sonoelastographic imaging of the PG seems particularly demanding. Several limitations have to be taken into account (36). Firstly, it should be assumed that PG neoplasms compress the adjacent gland parenchyma. The organ as a whole can scarcely expand due to a surrounding fibrous capsule. This might influence the stiffness of the whole organ, which may alter the measurement values, depending on the size of the tumor. Hence, it should be expected that the SWV-values increase with the size of the tumor, which was not the case according to our results (Figure 3, Table 3). Secondly, the expansion of the gland is further limited by its close contact with the mandible. The latter itself may further distort the measurement due to its bony structure, restricting the course, intensity and transmission of shear waves. Thirdly, values obtained with older US devices might be affected by tissue pre-compression caused by the contact between the US transducer and the skin (37). Although newer technologies claim that results are more or less independent of this pre-compression, all mentioned influencing factors might accumulate when examining the PG, impeding precise data acquisition.

According to our present results, we can partially agree with Zhang's conclusion: we observed a heterogeneity of measurement values in our study as well (Figures 3,4, Tables 2,3), even though two devices with almost identical quantitative shear wave techniques were used. In addition, the obtained SWV-values were not consistently dependent on the tumor size (Figure 3, Table 3). Consequently, the discrepancies in the values we recorded cannot be explained either by different assessment methods (both quantitative) or by different sonoelastography techniques (both impulsetriggered and SMV-based).

Our results indicate that these heterogeneous values were either due to the different consistencies of the tumors actually present, existing even within the same tumor entity, or to other confounding effects.

Regardless of the reason, we were consistently unsuccessful both in differentiating various tumor entities from one another and in distinguishing benign from malignant masses using both US devices (Table 4). These findings support and in particular augment previous results $(24,29,35,38-40)$, while opposing others $(25,34,41,42)$.

In detail, the majority of the cited studies included more or less subjective or manufacturer dependent scores to evaluate the elastographic findings, limiting their comparability. According to our knowledge, only four studies reported on quantitative SMV values $(29,34,42,43)$.

Matsuzuka and co-workers (34) used the VTIQ technique (as we did when using the $\mathrm{S} 3000$ system) and reported on mean SWV-values of $4.24 \mathrm{~m} / \mathrm{s}$ for benign and $6.52 \mathrm{~m} / \mathrm{s}$ for malignant lesions, with a cut-off value of $4.83 \mathrm{~m} / \mathrm{s}$. The authors observed significant differences, 


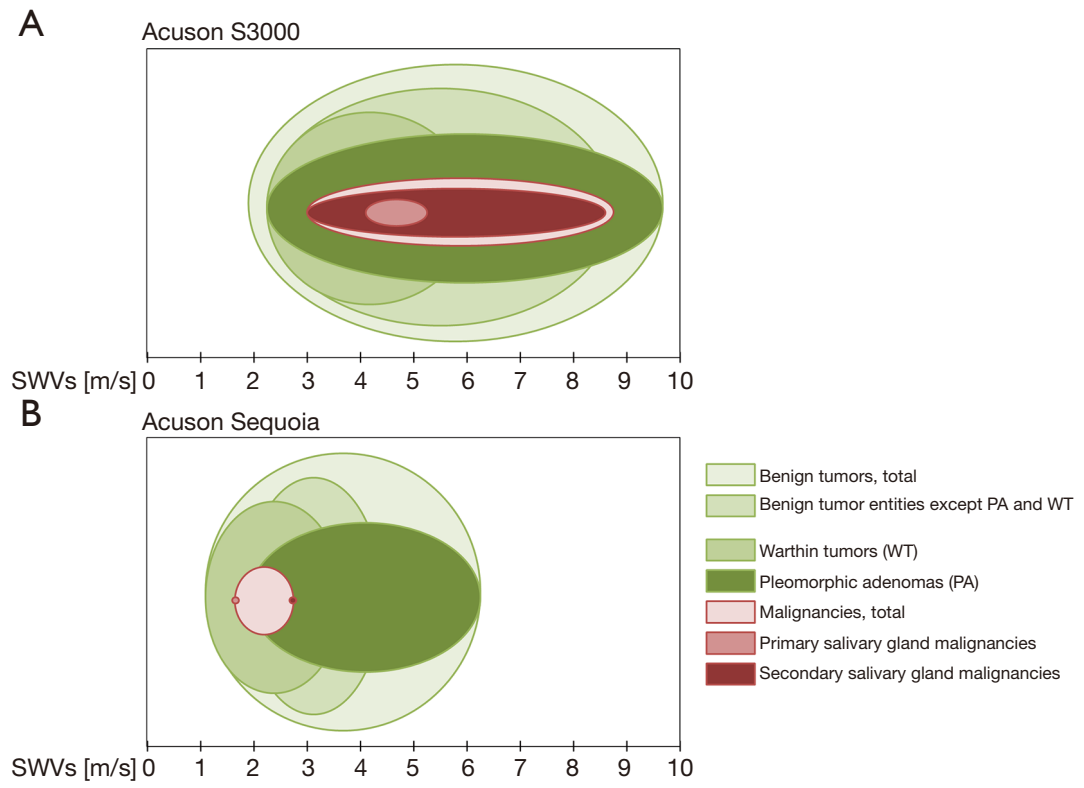

Figure 4 Illustration of shear wave velocity (SWV-)values obtained from different tumors of the parotid gland. The recorded values show a high degree of variation even within matching tumor entities, preventing a reliable differentiation from one another. The sizes of ellipses do not reflect the number of tumors. (A) SWV-values obtained with the Acuson S3000 system and (B) with the Acuson Sequoia system.

Table 4 Comparison of various tumor dignities/entities with one another, calculated for both ultrasound devices (Acuson S3000 and Acuson Sequoia)

\begin{tabular}{|c|c|c|c|c|}
\hline Differentiation & $P_{S 3000}$ & Spearman-Rho $_{\mathrm{S} 3000}$ & $P_{\text {Sequoia }}$ & Spearman-Rho Sequoia $_{\text {}}$ \\
\hline Benign except PA vs. malignant & 0.162 & 0.240 & 0.483 & 0.126 \\
\hline PA vs. WT & 0.001 & 0.492 & 0.067 & 0.324 \\
\hline PA vs. malignant & 0.270 & 0.277 & 0.234 & 0.249 \\
\hline
\end{tabular}

PA, pleomorphic adenomas; WT, Warthin tumors.

but the study only examined 11 benign and four malignant PG neoplasms. Matsuzuka's SWV-values are similar to our results (Table 2, S3000 system); however, we found neither significant differences nor a reliable cut-off value with respect to this specific question.

Zengel [51 tumors, Siemens Acuson S3000; (29)], Liu [76 tumors, Siemens Acuson S2000; (42)] and Yu [51 tumors, Siemens Acuson S2000; (43)] all used the VTQ technique, which recognizes $S W V$-values in direct proximity to the impulse beam in a preconditioned ROI. While Zengel reported no significant differences between benign and malignant lesions (SWV-values of $6.35 \pm 2.14$ and $6.24 \pm$ $2.91 \mathrm{~m} / \mathrm{s}$, respectively), Liu provided significantly different results for benign and malignant tumors (SWV-values of $2.14 \pm 0.25$ and $3.19 \pm 0.73 \mathrm{~m} / \mathrm{s}$, respectively; cut-off value of $2.45 \mathrm{~m} / \mathrm{s})$. Yu also listed significant differences in SWVvalues $(2.09 \pm 0.52 \mathrm{~m} / \mathrm{s}$ for benign and $2.94 \pm 0.39 \mathrm{~m} / \mathrm{s}$ for malignant masses; cut-off value $2.76 \mathrm{~m} / \mathrm{s}$ ). The comparison of all these results underlines the above-mentioned heterogeneity of the values, even when similar or identical techniques/devices or transducers were used.

We further realized that the spread of SWV-values obtained from different tumor entities when using one US system was much smaller than the spread of SWVvalues obtained from identical tumor entities in both of the two different US devices (Table 2). Neither a transfer 
of absolute SWV-values from one system to the other, nor a comparison between the two systems, nor establishing reliable general cut-off-values in both devices was consistently possible, emphasizing the system-dependence and tumor heterogeneity (Table 4).

The clinical impression exists that PAs are stiffer than other benign tumor entities. PAs exhibit different compositions of stromal and cellular components $(31,32)$, which may explain why the obtained measurement values range between those of other benign and malignant entities. Our results indicated that these variations in PA stiffness, however, were not solely responsible for the inability to differentiate benign from malignant lesions: even excluding the results obtained from PA from the analysis did not increase the discriminatory power of SWV-values (Figure 2A,B,C,D,E,F,G,H,I,f). The differences between PA and Warthin tumors were only significant when using the S3000 system (Table 4).

Our study is limited by the fact that every single tumor was examined with one US device only. It would have been desirable to obtain values from each tumor using both US devices to compare the measurements directly. This was not possible, as both US systems were not available at the same time in our department. Further, a large number of different PG tumor entities exist, not all of which can be represented in a single study due to the rareness of certain entities. The differentiation between benign and malignant lesions in general was moreover complicated by the fact that the considerable number of different tumor entities showed large spreads of SMV values, impeding the establishment of clear cut-off-values in our study (Table 2). Although the most common entities comprised the majority of cases in our cohort (PA and WT, $n=76 / 104,73.1 \%$ ), a reliable distinction between them was only possible with one (Acuson S3000), but not with the other device (Acuson Sequoia). Independent of the device or researcher, a reliable identification of the tumors was impossible, and clinical treatment decisions, as the overall goal, could not be made depending on this examination technique alone.

\section{Conclusions}

Taken together, a reliable differentiation or identification of PG tumors and clinical treatment decisions could not be made with the sole use of quantitative ARFIshear wave sonoelastography-independent of the device or researcher. If at all, the technique may serve as an additional examination tool to augment other US findings.
Conventional B-mode and color-Doppler imaging are still the US examination techniques of choice to evaluate salivary gland masses. As a future goal, the use of sonoelastographic techniques in the PG requires a sound standardization in order to be comparable between the different techniques and devices. Further refinements which can reliably detect minor differences are necessary to discriminate the different entities from one another.

\section{Acknowledgments}

Funding: None.

\section{Footnote}

Reporting Checklist: The authors have completed the MDAR reporting checklist. Available at http://dx.doi.org/10.21037/ gs-20-837

Data Sharing Statement: Available at http://dx.doi. org/10.21037/gs-20-837

Peer Review File: Available at http://dx.doi.org/10.21037/gs20-837

Conflicts of Interest: All authors have completed the ICMJE uniform disclosure form (available at http://dx.doi. org/10.21037/gs-20-837). The authors have no conflicts of interest to declare.

Ethical Statement: The authors are accountable for all aspects of the work in ensuring that questions related to the accuracy or integrity of any part of the work are appropriately investigated and resolved. Written informed consent was obtained from each patient for diagnostic procedures, therapeutic measures and scientific data processing, approved by the University's ethical review board (IRB No. 156_20 Bc), observing the university's general contract conditions and the World Medical Association Declaration of Helsinki (as revised in 2013).

Open Access Statement: This is an Open Access article distributed in accordance with the Creative Commons Attribution-NonCommercial-NoDerivs 4.0 International License (CC BY-NC-ND 4.0), which permits the noncommercial replication and distribution of the article with the strict proviso that no changes or edits are made and the original work is properly cited (including links to both the 
formal publication through the relevant DOI and the license). See: https://creativecommons.org/licenses/by-nc-nd/4.0/.

\section{References}

1. da Silva LP, Serpa MS, Viveiros SK et al. Salivary gland tumors in a Brazilian population: A 20-year retrospective and multicentric study of 2292 cases. J Craniomaxillofac Surg 2018;46:2227-33.

2. Pinkston JA, Cole P. Incidence rates of salivary gland tumors: results from a population-based study. Otolaryngol Head Neck Surg 1999;120:834-40.

3. Spiro RH. Salivary neoplasms: overview of a 35year experience with 2,807 patients. Head Neck Surg 1986;8:177-84.

4. Seethala RR, Stenman G. Update from the 4th Edition of the World Health Organization Classification of Head and Neck Tumours: Tumors of the Salivary Gland. Head Neck Pathol 2017;11:55-67.

5. Kato H, Kawaguchi M, Ando T, et al. CT and MR imaging findings of non-neoplastic cystic lesions of the parotid gland. Jpn J Radiol 2019;37:627-35.

6. Abdel Razek AAK, Mukherji SK. State-of-the-Art Imaging of Salivary Gland Tumors. Neuroimaging Clin N Am 2018;28:303-17.

7. Lobo R, Hawk J, Srinivasan A. A Review of Salivary Gland Malignancies: Common Histologic Types, Anatomic Considerations, and Imaging Strategies. Neuroimaging Clin N Am 2018;28:171-82.

8. Habermann CR, Arndt C, Graessner J, et al. Diffusionweighted echo-planar MR imaging of primary parotid gland tumors: is a prediction of different histologic subtypes possible? AJNR Am J Neuroradiol 2009;30:591-6.

9. Bozzato A, Zenk J, Greess H, et al. Potential of ultrasound diagnosis for parotid tumors: analysis of qualitative and quantitative parameters. Otolaryngol Head Neck Surg 2007;137:642-6.

10. Elmokadem AH, Abdel Khalek AM, Abdel Wahab RM, et al. Diagnostic Accuracy of Multiparametric Magnetic Resonance Imaging for Differentiation Between Parotid Neoplasms. Can Assoc Radiol J 2019;70:264-72.

11. Munhoz L, Ramos EADA, Im DC, et al. Application of diffusion-weighted magnetic resonance imaging in the diagnosis of salivary gland diseases: a systematic review. Oral Surg Oral Med Oral Pathol Oral Radiol 2019;128:280-310.

12. Zbären P, Triantafyllou A, Devaney KO, et al. Preoperative diagnostic of parotid gland neoplasms: fine-needle aspiration cytology or core needle biopsy? Eur Arch Otorhinolaryngol 2018;275:2609-13.

13. Mansour N, Bobenstetter L, Mansour S, et al. Differentiation of ultrasonographic hypoechoic head and neck lesions. Laryngorhinootologie 2019;98:701-7.

14. Kong X, Li H, Han Z. The diagnostic role of ultrasonography, computed tomography, magnetic resonance imaging, positron emission tomography/ computed tomography, and real-time elastography in the differentiation of benign and malignant salivary gland tumors: a meta-analysis. Oral Surg Oral Med Oral Pathol Oral Radiol 2019;128:431-43.e1.

15. Cantisani V, David E, Sidhu PS et al. Parotid Gland Lesions: Multiparametric Ultrasound and MRI Features. Ultraschall Med 2016;37:454-71.

16. Schick S, Steiner E, Gahleitner A, et al. Differentiation of benign and malignant tumors of the parotid gland: value of pulsed Doppler and color Doppler sonography. Eur Radiol 1998;8:1462-7.

17. Evans A, Whelehan P, Thomson K, et al. Differentiating benign from malignant solid breast masses: value of shear wave elastography according to lesion stiffness combined with greyscale ultrasound according to BI-RADS classification. Br J Cancer 2012;107:224-9.

18. Cantisani V, David E, Grazhdani H, et al. Prospective Evaluation of Semiquantitative Strain Ratio and Quantitative 2D Ultrasound Shear Wave Elastography (SWE) in Association with TIRADS Classification for Thyroid Nodule Characterization. Ultraschall Med 2019;40:495-503.

19. Esfahanian F, Aryan A, Ghajarzadeh M, et al. Application of Sonoelastography in Differential Diagnosis of Benign and Malignant Thyroid Nodules. Int J Prev Med 2016;7:55.

20. Kyriakidou G, Friedrich-Rust M, Bon D, et al. Comparison of strain elastography, point shear wave elastography using acoustic radiation force impulse imaging and 2D-shear wave elastography for the differentiation of thyroid nodules. PLoS One 2018;13:e0204095.

21. Lefebvre T, Wartelle-Bladou C, Wong P, et al. Prospective comparison of transient, point shear wave, and magnetic resonance elastography for staging liver fibrosis. Eur Radiol 2019;29:6477-88.

22. Sugimoto K, Moriyasu F, Oshiro H, et al. Clinical utilization of shear wave dispersion imaging in diffuse liver disease. Ultrasonography 2020;39:3-10.

23. Mahdavi SS, Moradi M, Wen X, et al. Evaluation of 
visualization of the prostate gland in vibro-elastography images. Med Image Anal 2011;15:589-600.

24. Altinbas NK, Gundogdu Anamurluoglu E, Oz II, et al. Real-Time Sonoelastography of Parotid Gland Tumors. J Ultrasound Med 2017;36:77-87.

25. Cantisani V, David E, de Virgilio A, et al. Prospective evaluation of Quasistatic Ultrasound Elastography (USE) compared with Baseline US for parotid gland lesions: preliminary results of elasticity contrast index (ECI) evaluation. Med Ultrason 2017;19:32-8.

26. Karaman CZ, Başak S, Polat YD et al. The Role of Real-Time Elastography in the Differential Diagnosis of Salivary Gland Tumors. J Ultrasound Med 2019;38:1677-83.

27. Klintworth N, Mantsopoulos K, Zenk J et al. Sonoelastography of parotid gland tumours: initial experience and identification of characteristic patterns. Eur Radiol 2012;22:947-56.

28. Mansour N, Bas M, Stock KF et al. Multimodal Ultrasonographic Pathway of Parotid Gland Lesions. Ultraschall Med 2017;38:166-73.

29. Zengel P, Notter F, Clevert DA. Does acoustic radiation force elastography improve the diagnostic capability of ultrasound in the preoperative characterization of masses of the parotid gland? Dentomaxillofac Radiol 2018;47:20180068.

30. Zhang YF, Li H, Wang XM et al. Sonoelastography for differential diagnosis between malignant and benign parotid lesions: a meta-analysis. Eur Radiol 2019;29:725-35.

31. Olgun DC, Kantarci F, Taskin U, et al. Relative proportions of stromal to cellular components of pleomorphic adenomas: determination with shear wave elastography. J Ultrasound Med 2014;33:503-8.

32. Seifert G, Langrock I, Donath K. Pathomorphologische Subklassifikation der pleomorphen Speicheldrüsenadenome. Analyse von 310 pleomorphen Parotisadenomen. HNO 1976;24:415-26.

33. Mansour N, Stock KF, Chaker A, et al. Evaluation of parotid gland lesions with standard ultrasound, color

Cite this article as: Thimsen V, Goncalves $M$, Koch $M$, Mantsopoulos K, Hornung J, Iro H, Schapher M. The current value of quantitative shear wave sonoelastography in parotid gland tumors. Gland Surg 2021;10(4):1374-1386. doi: 10.21037/gs20-837 duplex sonography, sonoelastography, and acoustic radiation force impulse imaging - a pilot study. Ultraschall Med 2012;33:283-8.

34. Matsuzuka T, Suzuki M, Saijo S et al. Stiffness of salivary gland and tumor measured by new ultrasonic techniques: Virtual touch quantification and IQ. Auris Nasus Larynx 2015;42:128-33.

35. Zengel P, Notter F, Clevert DA. VTIQ and VTQ in combination with B-mode and color Doppler ultrasound improve classification of salivary gland tumors, especially for inexperienced physicians. Clin Hemorheol Microcirc 2018;70:457-66.

36. Bruno C, Minniti S, Bucci A et al. ARFI: from basic principles to clinical applications in diffuse chronic disease-a review. Insights Imaging 2016;7:735-46.

37. Mantsopoulos K, Klintworth N, Iro H, et al. Applicability of shear wave elastography of the major salivary glands: values in healthy patients and effects of gender, smoking and pre-compression. Ultrasound Med Biol 2015;41:2310-8.

38. Bhatia KSS, Cho CCM, Tong CSL, et al. Shear wave elastography of focal salivary gland lesions: preliminary experience in a routine head and neck US clinic. Eur Radiol 2012;22:957-65.

39. Dumitriu D, Dudea S, Botar-Jid C, et al. Real-time sonoelastography of major salivary gland tumors. AJR Am J Roentgenol 2011;197:W924-30.

40. Westerland O, Howlett D. Sonoelastography techniques in the evaluation and diagnosis of parotid neoplasms. Eur Radiol 2012;22:966-9.

41. Heřman J, Sedláčková Z, Vachutka J, et al. Differential Diagnosis of Parotid Gland Tumors: Role of Shear Wave Elastography. Biomed Res Int 2017;2017:9234672.

42. Liu G, Wu S, Liang X, et al. Shear Wave Elastography Improves Specificity of Ultrasound for Parotid Nodules. Ultrasound Q 2018;34:62-6.

43. Yu J, Du Y, Lu Y, et al. Application of DTI and ARFI imaging in differential diagnosis of parotid tumours. Dentomaxillofac Radiol 2016;45:20160100. 